\title{
Challenging iatrogenic menopause. Oncofertility programme in Poland - a single-centre experience
}

\author{
Anna Horbaczewska ${ }^{1}$, Robert Jach ${ }^{1}$, Andrzej Zmaczyński ${ }^{1}$, Olga Kacalska-Janssen ${ }^{1}$, \\ Katarzyna Doroszewska ${ }^{1}$, Magdalena Piróg${ }^{1}$, Monika Kabzińska-Turek² \\ ${ }^{1}$ Clinic of Gynaecological Endocrinology, Jagiellonian University Medical College, Krakow, Poland \\ ${ }^{2}$ Clinic of Gynaecological Oncology, Jagiellonian University Medical College, Krakow, Poland
}

\begin{abstract}
Introduction: Menopause is defined as a 12-month period of time when menstruation permanently ceases. In some cases, menopause may be caused by external factors - for example gonadotoxic treatment that irreversibly damages ovarian tissue leading to loss of its hormonal and reproductive function. Oncofertility is a discipline that merges oncology and reproductive medicine, giving patients a chance to experience parenthood after gonadotoxic treatment is finished.

Aim of the study: The purpose of the study is to present the implementation and first outcomes of the Oncofertility Programme in the University Clinic of Endocrinological Gynaecology and Gynaecology, University Hospital in Krakow, Poland.

Material and methods: Patients interested in fertility preservation have been consulted in the University Clinic of Endocrinological Gynaecology and Gynaecology in Krakow since April 2016. Preliminary qualification to one of the available methods (embryo cryopreservation, oocyte cryopreservation, ovarian tissue cryopreservation) was conducted. Patients declaring a wish to join the programme were then referred to one of the three infertility treatment centres cooperating with the University Clinic, in order to undergo the chosen procedure.

Results: During a period of 24 months, 18 patients were consulted. The youngest consulted patient was 20 years old, the oldest 39. Two years after the first consultation, a telephone survey among consulted patients was carried out to verify whether the patients finally underwent oncofertility procedures, and to ask about their reproductive status.

Conclusions: The problem of fertility issues being inadequately addressed results in low referral rates to oncofertility programmes. Attempts to raise awareness of oncofertility possibilities among oncologists should be undertaken because critically few patients are being referred to oncofertility centres.
\end{abstract}

Key words: iatrogenic menopause, fertility preservation, cancer treatment, oncofertility.

\section{Introduction}

Menopause is defined as a 12-month period of time when menstruation permanently ceases, and it physiologically occurs between 45 and 55 years of age. However, in some cases menopause may be caused by external factors - for example, gonadotoxic treatment that damages ovarian tissue leading to irreversible loss of its hormonal and reproductive function. Patients exposed to treatment that may impair reproductive function are most frequently oncological patients [1].

Different types of cancer dominate in different age groups. Among young adults, i.e. 25-44 years old, women are most frequently diagnosed with breast cancer (45\%), melanoma (9\%), and cervical cancer (8\%) [2].
Advances in cancer treatment allow patients to be cured and live longer. Survival rates are known to be highest for patients aged between 15 and 44 years of age, with five-year survival ranging from $60 \%$ to $82 \%$ according to age, tumour site, and country of treatment. Nevertheless, cancer therapies often come with undesirable side effects. Among these, infertility may affect up to $80 \%$ of cancer survivors [3].

Oncofertility, a new discipline that merges oncology and reproductive medicine, gives adolescent and young adult patients a chance to experience the joy of parenthood after the treatment is finished.

Oncofertility was first named in 2015 by the American Society of Reproductive Medicine. The initiator and promoter of the discipline is Prof. Theresa Woodruff, the 
Dean and Associate Provost for Graduate Education in the Graduate School at Northwestern University.

The aim of this study is to present the process of implementation and first outcomes of the Oncofertility Programme in the University Clinic of Endocrinological Gynaecology and Gynaecology, University Hospital in Krakow, Poland.

\section{Material and methods}

Patients interested in fertility preservation, before the treatment that may impair reproductive potential, have been consulted in the University Clinic of Endocrinological Gynaecology and Gynaecology, University Hospital in Krakow since April 2016. Information about diagnosis, planned treatment, procreative plans, and lab results were obtained. Patients were informed about available fertility preservation procedures. Based on the clinical picture and patient's preference, preliminary qualification to one of the available methods (embryo cryopreservation, oocyte cryopreservation, ovarian tissue cryopreservation) was conducted. Patients declaring a wish to join the programme were then referred to one of the three infertility treatment centres cooperating with the clinic, in order to undergo the chosen oncofertility procedure. This is due to the fact that these kinds of procedures can only be conducted commercially because they are not covered by national health insurance according to Polish regulations. The study obtained Institutional Review Board consent in June 2015 (no. 122.6120.94.2015 and 122.6120.95.2015).

\section{Results}

During a period of 24 months, 18 patients were consulted. Diagnoses included oncological diseases: six cases of ovarian cancer (33\%), three cases of breast cancer (17\%), three cases of Hodgkin lymphoma (17\%), and one of cervical cancer (5\%); rheumatological diseases: one case of systemic lupus erythromatosus (SLE) (5\%),

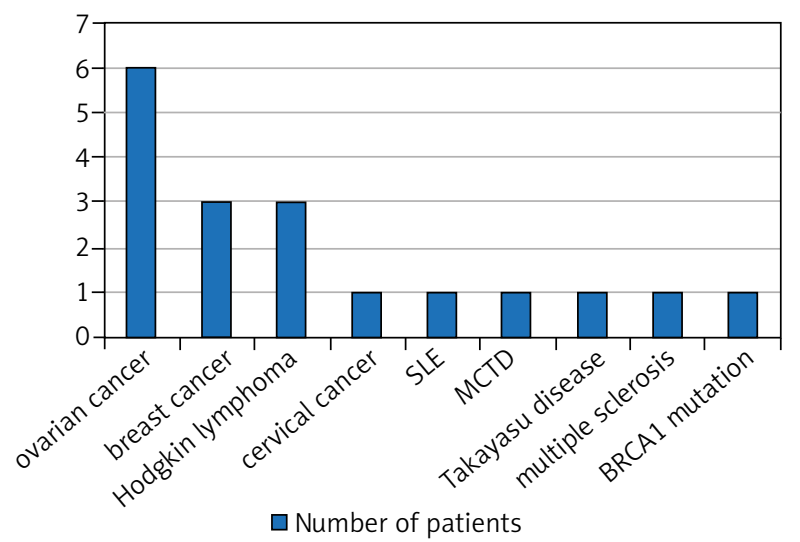

Fig. 1. Diagnoses of the consulted patients one mixed connective tissue disease (MCTD) (5\%), one Takayasu disease (5\%), as well as one case of multiple sclerosis (5\%) - the patient before myeloablation due to planned haematopoietic stem cell transplantation and one patient (5\%) with BRCA1 gene mutation (Fig. 1).

The youngest consulted patient was 20 years old, the oldest was 39. Mean age was 31 years, and the median value was 34 years.

Patients were referred to the Clinic by different specialists: six (33\%) by oncologist, four (22\%) by gynaecologist, three (17\%) by gynaecological oncologist, two (11\%) by internist, one (5\%) by rheumatologist, one $(5 \%)$ by haematologist, and one (5\%) by neurologist (Fig. 2).

Two of the consulted patients (11\%) had procreative plans at the time of consultation. One patient (5\%) already had children.

Time from diagnosis to oncofertility consultation differed between patients from two days to four years in one case. Mean time was 154 days, median value 25 days.

\section{Preliminary qualifications}

Until March 2018, 11 patients (61\% of the consulted patients) were qualified to the programme. Initially five patients ( $45 \%$ of the included to the programme) chose oocyte cryopreservation as a fertility preservation method because they did not have a partner. Three (27\%) patients opted for ovarian tissue cryopreservation due to contraindications for hormonal stimulation or lack of partner, two (18\%) couples initially chose embryo cryopreservation as the most effective fertility preservation method [4].

Four patients ( $22 \%$ of the consulted) did not express the wish to be included to the programme, in one case $(5 \%)$ fertility preservation was contraindicated due to medical reasons, and two patients were lost to follow-up.

Two years after the first consultation, a telephone survey among all consulted patients was carried out in

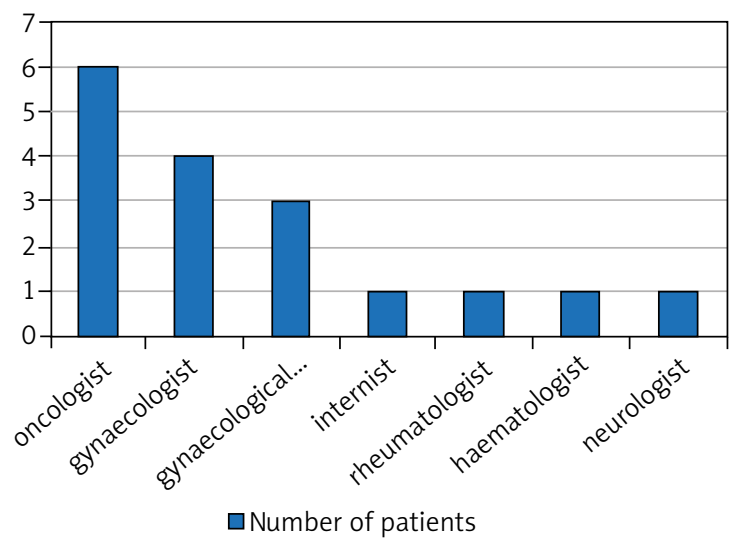

Fig. 2. Specialists referring patients for consultations 
order to verify whether the patients initially included in the programme finally decided to undergo fertility preserving procedures, ask about patients' current reproductive plans, and monitor their general health condition.

From 18 patients, two remained unavailable despite several attempts to contact them.

\section{Follow-up}

Four patients ultimately underwent oncofertility procedures (Fig. 3). Three of them were qualified for oocyte cryopreservation (because they did not have a partner at the time) and one for ovarian tissue cryopreservation because the immediate onset of the treatment was required.

One patient with cervical cancer underwent fertility-sparing surgery (radical laparoscopic trachelectomy with pelvic lymphadenectomy). No subsequent treatment was required. The patient has remained asymptomatic for 1.5 years and is currently planning to get pregnant.

None of the patients is planning to utilise preserved material in the forthcoming six months.

One patient did not undergo initially planned embryo cryopreservation at that time because state donation for in vitro fertilisation procedures ceased and the patient could not afford to cover the expenses associated with IVF.

Two of the patients consulted in the Clinic had already delivered healthy babies. None of these patients had undergone oncofertility procedures. Both conceived spontaneously during remission of the disease. Both pregnancies had undisturbed course.

\section{Efficacy of oncofertility consulting}

Time from diagnosis to oncofertility consultation in our Clinic ranged from two days to four years in one case (patient with Hodgkin lymphoma, after chemotherapy, not included to the programme due to documented loss of fertility). After exclusion of that case in the statistics, mean time from diagnosis to oncofertility consultation was 55 days, and the median value was 23 days.

All of the patients were referred to the infertility treatment centres on the day of the consultation in the Clinic. The time from first oncofertility consultation to oocyte retrieval varied from 25 to 63 days, mean time was 45 days. The patient qualified for ovarian tissue cryopreservation had the sample retrieved seven days after the consultation. The time from fertility preservation procedure to onset of the treatment varied from three to six days, mean time five days.

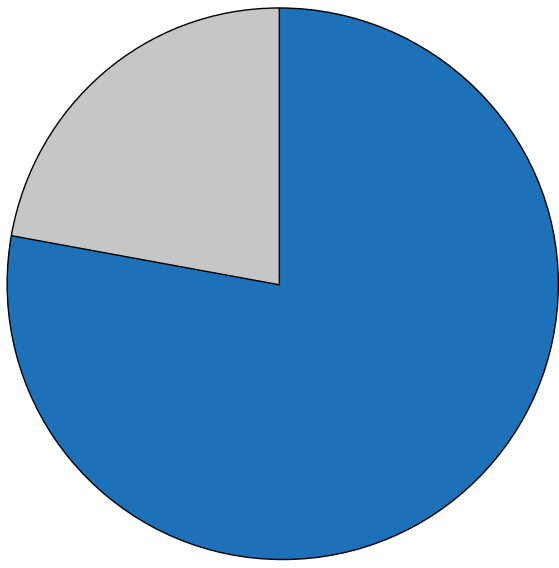

$\square$ Consulted patients who did not undergo oncofertility procedures $\square$ Patients who underwent oncofertility procedures

Fig. 3. Number of patients who finally underwent oncofertility procedures

All the patients underlined their positive attitude towards the consultations, regardless of whether they were included in the programme and finally underwent oncofertility procedures. As all of them claim that taking into consideration aspects of fertility while facing a serious diagnosis helped reduce stress and improve their general attitude towards treatment and vision for overcoming the disease. Two patients who could not be included in the programme for medical reasons expressed regretted that they were not informed about fertility-sparing options while it was still possible to perform them.

The most frequently reported drawback of the programme concerned the financial aspect of the fertility preservation because all the costs associated with fertility preservation procedures had to be covered by the patient.

\section{Discussion}

Based on current medical standards, the following methods of fertility preservation are available for women: embryo cryopreservation, oocyte cryopreservation, ovarian tissue cryopreservation, ovarian transposition, hormonal suppression, and fertility sparing surgery.

Methods of choice are embryo cryopreservation and oocyte cryopreservation. Both require undergoing hormonal stimulation in order to retrieve mature oocytes and optionally perform in vitro fertilisation. In particular medical conditions hormonal stimulation may be contraindicated (i.e. hormonal sensitive cancers) or an immediate onset of therapy may be necessary, not leaving time for hormonal stimulation $[5,6]$. Nevertheless, there are reports of beneficial effect of letrozole and tamoxifen during hormonal stimulation in breast cancer patients [7-11]. 
Ovarian tissue cryopreservation is still an experimental method. This procedure can be offered only as part of a clinical trial. It can be implicated in girls in prepubertal age and does not require a delay in the onset of therapy [12].

Operative ovarian transposition outside the smaller pelvis may be considered in patients before planned radiation on this region, and it should be performed as close to the beginning of the treatment as possible, due to the risk of dislocation of the ovaries [13].

In selected patients requiring surgical treatment the extent of surgery may be adjusted to the patient's procreative plans.

Hormonal suppression is not routinely recommended because its effectiveness remains controversial [14, 15].

The problem of fertility issues being inadequately addressed results in low referral rates to oncofertility programmes. This problem is reported by authors worldwide [16, 17]. However, in a single oncofertility centre in Milan a total of 96 patients were consulted during a period of three years [16]. A report from Gifu Prefecture in Japan details 81 patients consulted in an oncofertility clinic in Gifu University Hospital during a two-year period, referred from the 35 hospitals providing cancer treatment in that region [17]. As the report shows, the number of referred patients is constantly growing, indicating an increasing awareness of the aspect of future fertility among oncologists.

The reason why such a small number of patients is being consulted in our Clinic may be due to the fact that some patients are referred straight to the fertility treatment centres, omitting consultation in our Clinic. This, however, deprives the patient of the option to choose ovarian tissue cryopreservation as a fertility-preserving method. It can only be performed as part of a registered clinical trial, as it is in our Clinic.

As reports show, there are several obstacles on the path to fertility preservation. The most frequently mentioned barrier is the financial aspect of the procedure. In Poland it is not covered by national health insurance and requires an approximate expense of several hundred zloty (over 5000 USD). In the overwhelming majority of countries, with the exception of Denmark, France, Germany, the Netherlands, and Spain in Europe and Canada, Panama, and Argentina worldwide, oncofertility procedures are not covered by national health insurance [18].

A noticeably impaired aspect shown in our study is the time from diagnosis to oncofertility consultation. According to all oncofertility standards and recommendations, it should be organised as soon as possible after diagnosis. In our Clinic the time ranged from two days to four years in one case. After exclusion of that case in the statistics, mean time from diagnosis to oncofertility consultation was 55 days, and the median value was 23 days.
Worldwide, most patients (35\%) are referred to oncofertility specialist within 1-2 days of diagnosis. $78 \%$ see the specialist within one week [18].

In our Clinic the mean time from fertility preservation consultation to fertility preservation ranged from 25 to 63 days. Worldwide, most patients have fertility preservation procedures performed within 1-5 days (46\%), 83\% within two weeks.

Cancer treatment is a priority, and the therapy should not be delayed. Oncologists should have the decisive vote on whether to postpone the onset of cancer treatment because of fertility-sparing procedures [17]. Therefore, it is essential to start cancer treatment as soon as possible after preserving fertility. In our Clinic the time from fertility preservation to onset of the treatment varied from three to six days, with mean time of five days. Most patients worldwide start their treatment within 3-5 days after fertility preservation (27\%), $67 \%$ of them within one week [18].

The fact that draws attention is that although many patients are consulted for the possibility of preserving fertility, finally only a minority of them undergo these procedures. In our Clinic this comprised just four patients out of 18 consulted (22\%). Similar results were observed in Gifu Prefecture in Japan, where $70 \%$ of the patients consulted in an oncofertility centre finally did not decide to undergo fertility-sparing procedures [17]. This implies that making decisions about fertility preservation is a complex process and numerous external factors, e.g. clinical context, may have an influence.

\section{Future studies or recommendations}

Subsequent studies should include multicentre analysis and longer observation time because this will provide more objective data.

Constant discussion with oncologists and other specialists is necessary to spread awareness of the significance for the patient of the aspect of fertility preservation. It should be stressed that the oncological treatment is always a priority.

\section{Strengths and limitotions of the study}

The study presents observations of newly introduced type of health care service. This early analysis may act as a reference for other centres providing medical assistance in the field of oncofertility.

The presented study is not free from points that need to be further developed. More patients should be involved in the analysis to provide more adequate evaluation of performance of the programme. Furthermore, the time horizon of the analysis limits the spectrum of obstetrical observations. 


\section{Conclusions}

Cancer treatment is increasingly effective, enabling patients to live longer. Therefore, loss of fertility becomes a serious adverse effect of the therapy for young patients. Fortunately, reproductive medicine offers various methods of preserving fertility for the time that the treatment is completed. What is essential for the success of fertility sparing is timely referral from oncologist to an oncofertility centre, before cancer treatment is started.

Attempts to raise awareness of oncofertility possibilities among oncologists and other specialists should be undertaken because critically few patients are being referred to oncofertility centres. Once the patient is qualified for fertility preservation, further procedures are performed efficiently.

\section{Disclosure}

The authors report no conflict of interest.

\section{References}

1. Didkowska J, Wojciechowska U. Malignant Tumour Morbidity and Mortality in Poland. The National Cancer Registry, Oncology Centre - Maria Skłodowska-Curie Institute. http://onkologia.org.pl/ nowotwory-zlosliweogolem-2 (access: May 11, 2015).

2. United Kingdom Office for National Statistics on request, czerwiec 2014. http://www.ons.gov.uk/ons/rel/vsob1/cancer-statistics-registrations-england-series-mb1-/index.html (access: February 16, 2016).

3. Linkeviciute A, Boniolo G, Chiavari L, Peccatori FA. Fertility preservation in cancer patients: The global framework. Cancer Treat Rev 2014; 40: 1019. 1027.

4. Dolmans MM. Recent advances in fertility preservation and counseling for remale cancer patients. Expert Rev Anticancer Ther 2018; 18: 115-120.

5. Jeruss JS, Woodruff TK. Preservation of fertility in patients with cancer. N Engl J Med 2009; 360: 902-911.

6. De Vos M, Smitz J, Woodruff TK. Fertility preservation in women with cancer. Lancet 2014; 384: 1302-1310.

7. Azim AA, Costantini-Ferrando M, Oktay K. Safety of fertility preservation by ovarian stimulation with letrozole and gonadotropins in patients with breast cancer: a prospective controlled study. J Clin Oncol 2008; 26: $2630-2635$

8. Oktay K, Buyuk E, Libertella N, et al. Fertility preservation in breast cancer patients: a prospective controlled comparison of ovarian stimulation with tamoxifen and letrozole for embryo cryopreservation. J Clin Oncol 2005; 23: 4347-4353.

9. Lee S, Oktay K. Does higher starting dose of FSH stimulation with letrozole improve fertility preservation outcomes in women with breast cancer? Fertil Steril 2012; 98: 961.e1-964.e1.

10. Oktay K, Hourvitz A, Sahin G, et al. Letrozole reduces estrogen and gonadotropin exposure in women with breast cancer undergoing ovarian stimulation before chemotherapy. J Clin Endocrinol Metab 2006; 91: 3885-3890.

11. Azim AA, Costantini-Ferrando M, Lostritto K, et al. Relative potencies of anastrozole and letrozole to suppress estradiol in breast cancer patients undergoing ovarian stimulation before in vitro fertilization. J Clin Endocrinol Metab 2007; 92: 2197-2200.

12. Meirow D, Levron J, Eldar-Geva T, et al. Pregnancy after transplantation of cryopreserved ovarian tissue in a patient with ovarian failure after chemotherapy. N Engl J Med 2005; 353: 318-321.
13. Jach R, Pabian W, Spaczynski R, et al. Recommendtions of the Fertility Preservation Working Gruop in Oncological, Hematological and Other Patients Treated With Gonadotoxic Therapies „ONCOFERTILITY” (GROF) of the Polish Society of Oncological Gynecology. J Adolesc Young Adult Oncol 2017; 6: 388-395.

14. Del Mastro L, Boni L, Michelotti A, et al. Effect of the gonadotropinreleasing hormone analogue triptorelin on the occurrence of chemotherapy-induced early menopause in premenopausal women with breast cancer: a randomized trial. JAMA 2011; 306: 269-276.

15. Loibl S, Gerber B. Gonadotropin-releasing hormone analogue for premenopausal women with breast cancer. JAMA 2011; 306: 1760, 1761.

16. Sigismondi C, Papaleo E, Viganò P, et al. Fertility preservation in female cancer patients: a single center experience. Chin J Cancer 2015; 34: 56-60.

17. Furui T, Takenaka M, Makino H, et al. An evaluation of the Gifu Model in a trial for a new regional oncofertility network in Japan, focusing on its necessity and effects. F Reprod Med Biol 2015; 15: 107-113.

18. Rashedi A, de Roo Saskia, Ataman L, et al. Survey of Third-Party Parenting Options Associated With Fertility Preservation Available to Patients With Cancer Around the Globe. J Glob Oncol 2018; 4: 1-7. 